UMD-PP-00-099

\title{
Neutrino Mass, Bulk Majoron and Neutrinoless Double Beta Decay
}

\author{
R. N. Mohapatra ${ }^{1 *}$, A. Pérez-Lorenzana ${ }^{1,2 \dagger}$ and C. A. de S. Pires ${ }^{1 \ddagger}$ \\ ${ }^{1}$ Department of Physics, University of Maryland, College Park, MD, 20742, USA \\ ${ }^{2}$ Departamento de Fúsica, Centro de Investigación y de Estudios Avanzados del I.P.N. \\ Apdo. Post. 14-740, 07000, México, D.F., México.
}

(August, 2000)

\begin{abstract}
A new economical model for neutrino masses is proposed in the context of the brane-bulk scenarios for particle physics, where the global $B-L$ symmetry of the standard model is broken spontaneously by a gauge singlet Higgs field in the bulk. This leads to a bulk singlet majoron whose Kaluza-Klein excitations may make it visible in neutrinoless double beta decay for some parameter range if the string scale is close to a $\mathrm{TeV}$.
\end{abstract}

14.60.Pq; 14.60.St.

\footnotetext{
*e-mail:rmohapat@physics.umd.edu

†e-mail:aplorenz@Glue.umd.edu

${ }^{\ddagger}$--mail:cpires@physics.umd.edu
} 


\section{INTRODUCTION}

One of the major phenomenological challenges for models with large extra dimensions and low string scale [1] is to understand the small mass of neutrinos. The basic problem arises due to the fact that the effective theory below the string scale will have nonrenormalizable operators which are suppressed by powers of the string scale $M$. The operator relevant for neutrino masses is of the form $L H L H / M$, where $L$ and $H$ are the lepton and Higgs doublets of the standard model respectively. After symmetry breaking, it leads to neutrino masses which are much too large. In general, higher dimensional operators can also create other phenomenological difficulties for such models e.g. rapid proton decay via operators of the form $Q Q Q L / M$; however, it has been suggested that operators that involve different matter fields such as the ones that lead to proton decay, can be suppressed by using the idea of "fat" branes [2] where different matter fields are located at different points in the brane. This idea, however, does not help in the case of the neutrino mass operator above since it involves only one matter field and since the Higgs field needs to be "spread out" rather than localized for all fermions to have mass. One must therefore seek other ways to suppress the effects of this operator.

A simple way to understand small neutrino masses in these models, suggested early on, is to assume the existence of a global $B-L$ symmetry and include only bulk neutrinos in addition to the standard model particles [3]. This leads to small neutrino masses for natural values of all parameters, due to suppressed overlap of the wave function between the brane and the bulk fields. The neutrinos in this model are Dirac particles. A second suggestion is to use a local $B-L$ symmetry [4], which generically requires the string scale to be intermediate rather than $\mathrm{TeV}$ type. The neutrinos in this model can be either Dirac or Majorana particles. By now, the phenomenology of the former case has been studied extensively in several papers [5].

More recently an alternative suggestion has been put forth [6] where the global $B-L$ symmetry of the standard model is assumed to be an exact symmetry of the complete model so that all undesirable higher dimensional terms contributing to neutrino mass are forbidden. However, instead of adding extra neutrinos to the bulk, a scalar field in a separate brane is used to break the $B-L$ symmetry spontaneously. This leads to a singlet majoron [7], which practically decouples from the theory even though the scales are very small. The smallness of neutrino masses in this model arise from the Yukawa like suppression (called "shining" [8]) that has its origin in the propagator of a massive bulk field (denoted by $\chi$ ). In order to implement this picture, one needs the number of large extra dimensions to be at least three, preferably more. The reason for at least three extra dimensions is that the desired suppression takes place only in these cases. Furthermore, four or more are preferable because in case of three extra dimensions, the relation

$$
M_{P \ell}^{2}=M^{5} R^{3},
$$

implies that the size of the extra dimension is $R \leq(\mathrm{KeV})^{-1}$ for $M \geq 1 \mathrm{TeV}$. Since to get small neutrino masses via the "shining" effect one needs $m_{\chi} \ll R^{-1}$, the bulk field must have a tiny mass, much less than a keV. If the number of extra dimensions is four or more, this constraint on the parameter $m_{\chi}$ becomes much weakened, requiring less "fine tuning" in the theory. The other point is that even for three extra dimensions, their sizes become so 
small that the current gravity experiments lose their usefulness as ways to search for their existence.

In this paper, we like to pursue the idea that spontaneous breaking of $B-L$ symmetry may indeed be the origin of neutrino masses in models with large extra dimensions but with sizes of extra dimensions in the millimeter range and furthermore, we will work only with one large extra dimension. As far as the string scale $M$ goes, we will assume that $M \sim 30$ TeV so as to satisfy the SN1987A bounds [9] for the case of one large extra dimension. Sizes of other dimensions will be accordingly adjusted so as to satisfy the generalized version of the relation in Eq. (1). In order to get nonvanishing neutrino masses, we will assume that the $B-L$ symmetry is spontaneously broken by a gauge singlet scalar field $\chi$ in the bulk. This scalar field $\chi$ which is assumed to carry two units of lepton number $(B-L)$, is the only extra field in the model, thus making it the most economical extension of the standard model to date that leads to neutrino masses. (Note in contrast that in the bulk neutrino alternative, one needs a minimum of three bulk fermions to get a realistic mass pattern).

An interesting feature of this model is that in some extreme domains of the parameter space of the model, the singlet majoron has a chance to be visible in processes such as neutrinoless double beta decay due to its many Kaluza-Klein excitations. A disadvantage is that to get neutrino masses in the $\mathrm{eV}$ range, some suppression of the strengths of the higher dimensional operators or a small value for $m_{\chi}^{2}$ is needed, unlike the bulk neutrino models. The required suppressions are at the same level as that needed for example in the triplet majoron model [10,11.

\section{BULK SINGLET AND NEUTRINO MASS}

Our scenario consists of standard model in the brane, to which we add only one gauge singlet complex scalar field $\chi$ propagating in the bulk. We assume that (i) the field $\chi$ carries two units of $B-L$ quantum number; (ii) the model respects global $B-L$ symmetry prior to symmetry breaking by vacuum and (iii) it includes in the Lagrangian operators of all dimensions that conserve $B-L$ symmetry. A list of some of the leading operators are:

$$
\mathcal{L}(x)=\int d y\left[\frac{f}{M^{5 / 2}}(L H)^{2} \chi(x, y)+\frac{f^{\prime}}{M^{17 / 2}} Q^{6} \tilde{H}^{2} \chi^{*}(x, y)\right] \delta(y),
$$

where $\tilde{H}=i \tau_{2} H^{*}$. We have not included any operator that could be suppressed by appropriate "fattening" of the brane [2]. The second operator in Eq. (2) is also not suppressed in the fat brane scenario and is a $\Delta B=2$ operator that can give rise to the process of neutron-anti-neutron oscillation [12]. We will discuss this later.

In order to implement spontaneous breaking of $B-L$ symmetry, let us write down the bulk scalar potential for $\chi$. The part of the potential important here is

$$
V(\chi)=-\frac{m_{\chi}^{2}}{2} \chi^{\dagger} \chi+\frac{\lambda}{4 M}\left(\chi^{\dagger} \chi\right)^{2}
$$

Minimizing this potential, we find that at its minimum, the singlet field has the vev

$$
\langle\chi\rangle_{B}=\frac{m_{\chi} M^{1 / 2}}{\sqrt{\lambda}}
$$


Using this, we find that after electroweak symmetry breaking i.e. $\langle H\rangle=v_{w k}$, the neutrinos acquire a Majorana mass given by:

$$
m_{\nu}=\frac{f}{M^{5 / 2}} v_{w k}^{2}\langle\chi\rangle
$$

For $M=30 \mathrm{TeV}$, we can generate neutrino mass of order of $\mathrm{eV}$ if we take $\langle\chi\rangle=5 \times\left(\frac{10^{-3}}{f}\right)$ $\mathrm{GeV}^{3 / 2}$. This leads to $m_{\chi} \simeq 90 \times\left(\frac{10^{-3}}{f}\right) \mathrm{MeV}$ (for $\lambda \sim 10$ ). Thus we need somewhat of a strong fine tuning of the parameters of the bulk fields to get the right order for neutrino masses. This fine tuning is at the same level as that required in the case of the triplet majoron model [10]. Despite this feature, we consider these models to be of interest since they appear rather economical and embody a new phenomenon not hitherto discussed in the context of neutrino masses. Furthermore, the small $m_{\chi}^{2}$ values could perhaps be made natural if there is supersymmetry, while keeping the other features unaffected. As we discuss below, the extreme small mass range of $\chi$ has one advantage that it makes the associated goldstone boson, the majoron more visible in certain low energy processes.

It is clear that due to spontaneous breaking of $B-L$ symmetry in the bulk, this model has the massless particle, majoron (the CP-odd part of the singlet $\chi$, denoted by $J$ ), which is a bulk field. In four dimensions, the majoron has a tower of partners with masses separated by a tiny amount $\left(\sim 10^{-3} \mathrm{eV}\right)$ for millimeter extra dimensions. They will be produced as a whole tower in any process where majoron is produced. Furthermore, the real part of the field $\chi$ (to be denoted by $\sigma_{\chi}$ ) also has a mass $\frac{m_{\chi}^{2}}{2}$. Since in this model $m_{\chi}$ has a value in the range of few $\mathrm{MeV}$ or less, it and its tower could also be produced in processes that have enough phase space. We give the example of the neutrinoless double beta decay in the next section, where only the majoron is produced unless the $\sigma_{\chi}$ has a mass in the sub $\mathrm{MeV}$ range. In processes such as muon decay however, both particles will be produced although the amplitude for it is highly suppressed.

The neutrino mass texture in this model arises purely from the flavor profile of the higher dimensional coupling $f_{i j}$. Experimental data on neutrino oscillations will fix this profile. As an example, which embodies the so-called bimaximal neutrino mixing pattern and nearly degenerate neutrino masses, we provide the following $f$ matrix:

$$
f=\left(\begin{array}{ccc}
m_{0} & \frac{c s}{\sqrt{2}} \delta_{S} & \frac{c s}{\sqrt{2}} \delta_{S} \\
\frac{c s}{\sqrt{2}} \delta_{S} & m_{0}+\delta_{A} / 2 & \delta_{A} / 2 \\
\frac{c s}{\sqrt{2}} \delta_{S} & \delta_{A} / 2 & m_{0}+\delta_{A} / 2
\end{array}\right)
$$

where $m_{0}$ is the common mass, $c=\cos \theta$ and $s=\sin \theta ; \delta_{A, S}$ are responsible for the mass splittings that explain the atmospheric and solar neutrino data. For $c=s=\frac{1}{\sqrt{2}}$, we get the bimaximal pattern. An advantage of the mass degeneracy is that it enhances the contribution to the neutrinoless double beta decay.

\section{NEUTRINOLESS DOUBLE BETA DECAY WITH MAJORON EMISSION}

One of the primary experimental manifestation of the majoron idea is in the process of neutrinoless double beta decay with majoron emission, a fact which was first noted for the 
case of the triplet majoron [10] in ref. [11]. Note that the original singlet majoron coupling to neutrinos is so weak that it is generally not visible in this process. However, the bulk majoron, though a gauge singlet, is different. It can be produced in neutrinoless double beta decay via the diagram in Fig. 1.

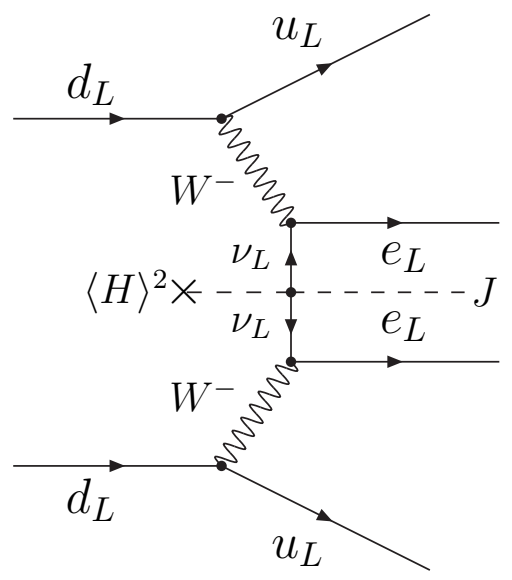

Fig. 1: Majoron emission in neutrinoless double beta decay in the bulk majoron model

The differential decay width for this process can be written as:

$$
\frac{d^{2} \Gamma}{d \epsilon_{1} d \epsilon_{2}}=A_{N u c l}^{2} \frac{G_{F}^{4} p_{F}^{2}}{8 \pi^{5}}\left(\frac{f^{2} v_{w k}^{4}}{M^{5}}\right)\left(E-\epsilon_{1}-\epsilon_{2}\right)^{2} \epsilon_{1} k_{1} \epsilon_{2} k_{2},
$$

where $\epsilon_{1,2}$ and $k_{1,2}$ are the electron energy and momenta respectively, $A_{N u c l}$ is a dimensionless nuclear factor, whose value we take from nuclear calculations for the single majoron decay mode [13]; $p_{F}$ is the Fermi momentum in nuclei (which we take to be $100 \mathrm{MeV}$ ); $E$ is the available energy for electrons and the majoron in the decay [13]. Note that there are two powers of the factor $\left(Q-\epsilon_{1}-\epsilon_{2}\right)$ (the power of this factor in the differential decay distribution is called in the literature as spectral index [14]) above in contrast with a single power in the triplet majoron model and generally odd powers in most theoretical models [15. This is the effect of the tower of majoron KK modes. The lower limits on the lifetime for the process $\beta \beta_{0 \nu J}$ from various nuclei are now at the level of $7.2 \times 10^{20}$ years for ${ }^{48} \mathrm{Ca}$ [16] to $7.2 \times 10^{21}$ yrs for ${ }^{130} \mathrm{Xe} \mathrm{[18]} \mathrm{and}{ }^{76} \mathrm{Ge}$ [17]. For $A_{N u c l} \sim 0.1$ and $f \sim 1$, using the best of the above experimental limits [13] for majoron emission in $\beta \beta_{0 \nu J}$, we get a lower limit on $M \geq 1 \mathrm{TeV}$. This bound can be improved once the search for the majoron emitting double beta decay is carried out at higher precision level by experiments such as for instance the proposed GENIUS 19] experiment as well as others.

\section{NEUTRON-ANTI-NEUTRON OSCILLATION}

Let us now turn to the second operator in Eq. (1), which leads to $\Delta B=2$ transitions. The strength of this operator is given by

$$
G_{\Delta B=2} \sim \frac{f^{\prime}\langle\chi\rangle_{B} v_{w k}^{2}}{M^{17 / 2}}
$$


For the values of $M \simeq 30 \mathrm{TeV}$ and $\langle\chi\rangle_{B}=10-0.01 \mathrm{GeV}^{3 / 2}$ discussed above, we get $G_{\triangle B=2} \sim 10^{-32}-10^{-35} \mathrm{GeV}^{-5}$. This translates into an oscillation time for $N-\bar{N}$ from $10^{10}$ to $10^{15}$ sec. after allowing for uncertainties in the hadronic matrix elements. The lower values are in the range accessible to a proposed experiment [20].

The same operator also leads to a novel process where an infinity tower of KK majorons are emitted in the transition $N N \rightarrow \chi$ or in terms of actual nuclear transmutation $(Z, A) \rightarrow$ $(Z, A-2)+\chi$. The width for this process is given by:

$$
\Gamma_{\Delta B=2} \sim M^{-17} m_{N}^{14} v_{w k}^{4} 10^{-6} G e V
$$

where we have used the factor of $10^{-6}$ to denote the hadron dressing of the six quark operator. For $M=30 \mathrm{TeV}$, the nuclear instability life times implied by this are $\sim 10^{39}$ yrs. However, if we ignored other constraints on $M$ and chose it to be of order $10 \mathrm{TeV}$ or so, we would expect majoron emitting modes of the above type with a life time of about $10^{32}$ years which looked for perhaps even in existing data.

\section{CONCLUSION}

In this brief note, we have suggested a new model for neutrino masses in theories with large extra dimensions using spontaneous breaking of lepton number symmetry by a bulk scalar field. The resulting bulk majoron may be visible in neutrinoless double beta decay experiments for certain domains of parameters, if the string scale is indeed close to a $\mathrm{TeV}$. The model also predicts a novel baryon number violating process where two neutrons in a nucleus disappear with the emission of a majoron which would lead to missing energy proton decay events.

Acknowledgements. The work of RNM is supported by a grant from the National Science Foundation under grant number PHY-9802551. The work of APL is supported in part by CONACyT (México). The work of CP is supported by Fundação de Amparo à Pesquisa do Estado de São Paulo (FAPESP). 


\section{REFERENCES}

[1] N. Arkani-Hamed, S. Dimopoulos, G. Dvali, Phys. Lett. B429 (1998) 263.

[2] N. Arkani-Hamed and M. Schmaltz, Phys. Rev. D61 (2000) 033005.

[3] K. R. Dienes, E. Dudas and T. Gherghetta, Nucl. Phys. B557 (1999) 25; N. ArkaniHamed, S. Dimopoulos, G. Dvali and J. March-Russell, hep-ph/9811448; G. Dvali and A.Y. Smirnov, Nucl. Phys. B563 (1999) 63.

[4] R. N. Mohapatra, S. Nandi and A. Pérez-Lorenzana, Phys. Lett B466 (1999) 115; R. N. Mohapatra and A. Pérez-Lorenzana, Nucl. Phys. B576 (2000) 466.

[5] R. Barbieri, P. Creminelli and A. Strumia, hep-ph/0002199; A. Faraggi and M. Pospelov, Phys. Lett. B458 (1999) 237; G. C. McLaughlin, J. N. Ng, Phys. Lett. B470 (1999) 157; nucl-th/0003023; R. N. Mohapatra, A. Perez-Lorenzana, hep-ph/0006278; A. Das and O. Kong, Phys. Lett. B470 (1999) 149; A. Lukas and A. Romanino, hep-ph/0004130; Y. Grossman and M. Neubert, Phys. Lett. B474 (2000) 361; A. Lukas, P. Ramond, A. Romanino, G. G. Ross, hep-ph/0008049; A. Ionissian and A. Pilaftsis, hep-ph/9907522; A. Ionissian and J. W. F. Valle, hep-ph/9911349.

[6] E. Ma, M. Raidal, U. Sarkar, hep-ph/0006046; E. Ma, G. Rajasekaran and U. Sarkar, hep-ph/0006340.

[7] Y. Chikashige, R. N. Mohapatra and R. D. Peccei, Phys. Lett. B 98 (1981) 265.

[8] N. Arkani-Hamed, S. Dimopoulos, hep-ph/9811353.

[9] V. Barger, T. Han, C. Kao, R.J. Zhang, Phys. Lett. B461 (1999) 34

[10] G. Gelmini and M. Roncadelli, Phys. Lett. 99 (1980) 1411.

[11] H. Georgi, S. L. Glashow and S. Nussinov, Nucl. Phys. B193 (1981) 297.

[12] R. N. Mohapatra and R. E. marshak, Phys. Rev. Lett. 44 (1980) 1316.

[13] For a review, see J. Hellmig, M. Hirsch, H. Klapdor-Kleingrothaus, B. Maier and H. Paes, in Double Beta Decay and Related processes, ed. H. Klapdor-Kleingrothaus and S. Stoica, p. 130 (World Scientific, 1997) and for a theoretical review, see C. Burgess, ibid, p. 110. For a recent limit from the NEMO-2 experiment, see R. Arnold et al., ITEP preprint, $15 / 00$.

[14] C. Burgess and J. Cline, Phys. Lett. B298 (1993) 141.

[15] P. Bamert, C. Burgess and R. N. Mohapatra, Nucl. Phys. B449 (1995) 25.

[16] A. Barabash et al.Phys. Lett. B 216 (1989) 257.

[17] M. Gunther et al. Phys. Rev. D55 (1997) 54.

[18] R. Luesher et al. Phys. Lett. B434 (1998) 407.

[19] H. Klapdor-Kleingrothaus et al. hep-ex/9910205.

[20] Y. Kamyshkov, Proceedings of the International Workshop on Baryon Instability, ed. Y. Kamyshkov et al. Oak Ridge (1996). 